\title{
A Novel Therapeutic Strategy Combining Use of Intracellular Magnetic Nanoparticles under an Alternating Magnetic Field and Bleomycin
}

\author{
Yoshimi Inaoka1, Tamami Keii', Atsushi Mimura1, Kenya Murase 1,2* \\ ${ }^{1}$ Department of Medical Physics and Engineering, Division of Medical Technology and Science, Faculty of Health Science, \\ Graduate School of Medicine, Osaka University, Suita, Osaka, Japan \\ ${ }^{2}$ Global Center for Medical Engineering and Informatics, Osaka University, Suita, Osaka, Japan \\ Email: *murase@sahs.med.osaka-u.ac.jp
}

How to cite this paper: Inaoka, Y., Keii, T., Mimura, A. and Murase, K. (2019) A Novel Therapeutic Strategy Combining Use of Intracellular Magnetic Nanoparticles under an Alternating Magnetic Field and Bleomycin. Open Journal of Applied Sciences, 9, 87-103.

https://doi.org/10.4236/ojapps.2019.93008

Received: February 6, 2019

Accepted: March 10, 2019

Published: March 13, 2019

Copyright $\odot 2019$ by author(s) and Scientific Research Publishing Inc. This work is licensed under the Creative Commons Attribution International License (CC BY 4.0).

http://creativecommons.org/licenses/by/4.0/

(c) (i) Open Access

\begin{abstract}
Purpose: The purpose of this study was to present a novel therapeutic strategy combining use of intracellular magnetic nanoparticles (MNPs) under an alternating magnetic field (AMF) and bleomycin (BLM), and to evaluate its therapeutic effect using tumor-bearing mice. Materials and Methods: MNPs (Resovist $^{\oplus}, 1.05 \mathrm{mg}$ iron) were incorporated into the hemagglutinating virus of Japan-envelope (HVJ-E) vector $\left(\sim 5 \times 10^{9}\right.$ particles) (HVJ-E/MNPs) by centrifugation at $10,000 \times \mathrm{g}$ for $5 \mathrm{~min}$ at $4^{\circ} \mathrm{C}$. Tumor-bearing mice were prepared by inoculating Colon- 26 cells subcutaneously into the backs of BALB/C mice. When the tumor volume reached $\sim 100 \mathrm{~mm}^{3}$, HVJ-E/MNPs and/or BLM were injected directly into the tumor. The AMF was applied to the mice one hour after the injection of agents (AMF treatment). The mice injected with HVJ-E/MNPs were imaged using our magnetic particle imaging (MPI) scanner immediately (13 $\mathrm{min}$ ) before, immediately $(22 \mathrm{~min})$ after, and 3, 7, and 14 days after the injection of agents, and the temporal changes of the average and maximum MPI pixel values in the tumor were quantitatively evaluated. The therapeutic effect was evaluated by calculating the relative tumor volume growth (RTVG) from the tumor volumes measured each day. Transmission electron microscopic (TEM) observation of resected tumors was also performed to confirm the intracellular distribution of MNPs. Results: The AMF treatment combined with BLM significantly decreased the RTVG value compared with AMF treatment alone at 9 to 14 days, and BLM alone at 3 to 5 days after AMF treatment. The average and maximum MPI pixel values in the tumor were almost constant for 14 days. TEM observation confirmed that most of the HVJ-E/MNPs were internalized into tumor cells within one hour after injection. Conclusion: A novel therapeutic strategy with use of AMF
\end{abstract}


treatment and BLM was presented, and the time-dependent change of MNPs in tumors was evaluated using MPI. The present results suggest that this novel strategy can suppress tumor volume growth over AMF treatment or BLM alone, and can be performed repeatedly with a single injection of HVJ-E/MNPs. They also suggest that HVJ-E is effective for internalizing MNPs into cancer cells and that MPI allows for longitudinal monitoring of the distribution of MNPs in tumors.

\section{Keywords}

Magnetic Particle Imaging (MPI), Magnetic Nanoparticles (MNPs),

Hemagglutinating Virus of Japan-Envelope (HVJ-E), Intracellular Magnetic

Hyperthermia, Bleomycin (BLM)

\section{Introduction}

Hyperthermia is a well-established approach to cancer therapy, and is based on the fact that cancer cells are more sensitive to heat than normal tissues [1]. The therapeutic effect of hyperthermia depends on the temperature and heating duration; its efficacy increases dramatically at temperatures above $42.5^{\circ} \mathrm{C}$ [1]. However, conventional hyperthermia treatments such as the use of an external water bath and radio frequency-capacitive heating cannot easily heat deep-seated tumors [1].

Magnetic hyperthermia treatment employs magnetic nanoparticles (MNPs) as a source of localized heat [2]. MNPs heat through hysteresis loss and/or magnetic relaxation such as Néel and Brownian relaxation under an alternating magnetic field (AMF) [3]. In addition to their heating property under an AMF, MNPs have several biomedical advantages. They are suitable for drug delivery because their size is small enough to get close to biological entities, and their surface is easily modified with antibodies, polymers, and functionalized peptides [4]. Owing to their magnetic property, they can be manipulated by an external magnetic field to accumulate to targeted regions [5]. If MNPs can be delivered to tumors, magnetic hyperthermia can then selectively heat tumor cells without damaging normal tissues [6].

One of the optimal methods to selectively heat tumor cells would be the use of intracellular MNPs taken up by tumor cells, i.e., intracellular magnetic hyperthermia. The concept of intracellular magnetic hyperthermia was first proposed by Gordon et al. [7]. Intracellular magnetic hyperthermia showed a more potent cell-killing effect than heating in a water bath, and the use of functionalized MNPs provided a promising approach for intracellular magnetic hyperthermia [8]. Recently, we investigated the usefulness of intracellular magnetic hyperthermia in comparison with extracellular magnetic hyperthermia, and reported that intracellular magnetic hyperthermia is more cytotoxic than extracellular magnetic hyperthermia despite a lower temperature rise in tumors [9]. Thus, it 
is desirable to develop a method for facilitating the rapid internalization of as many MNPs as possible into cancer cells, in order to enhance the effectiveness of intracellular magnetic hyperthermia. To date, various methods for surface modification [4], and vectors including peptide vectors such as protamine [10], have been developed to realize the rapid internalization of MNPs into cells.

The hemagglutinating virus of Japan-envelope (HVJ-E) vector was developed as a versatile vehicle for gene delivery by Kaneda et al. [11]. Although it is originally a rodent pneumonia virus, it can be used as non-viral vector because the infectivity of this virus is inactivated by ultraviolet (UV) irradiation. The notable advantage of this novel vector is its capacity for cell membrane fusion, which allows efficient incorporation of encapsulated materials into cells regardless of cellular condition [11]. The conventional cellular uptake of MNPs mainly occurs through endocytosis or phagocytosis. Since such uptake pathways depend on the cellular condition, there is a tendency for the cellular uptake to take several hours and for its efficiency to be low [12]. With cell membrane fusion, the HVJ-E vector does not have such a problem and can introduce materials into cells quickly and efficiently. Thus, it has great potential for a variety of applications [12] [13] [14].

MNPs have potential not only as a heating source for hyperthermia but also as imaging agents for magnetic resonance imaging (MRI) or magnetic particle imaging (MPI). MPI was introduced as a new imaging method in 2005 [15], and allowed imaging of the spatial distribution of MNPs with high sensitivity and high spatial resolution [16] [17] [18]. MPI is superior to MRI in terms of the visibility of MNPs and quantitative performance, because MPI can image MNPs in positive contrast and its pixel value linearly correlates with the iron concentration of MNPs [18].

Bleomycin hydrochloride (BLM) is an anticancer antibiotic [19]. It is known that a combination of BLM and hyperthermia enhances the antitumor effect of BLM [20]. BLM has a unique mechanism of cytotoxicity that is caused by breaking the double helical structure of deoxyribonucleic acid (DNA) [21]. BLM is activated in the presence of $\mathrm{Fe}(\mathrm{II})$ ion and oxygen, which serve as cofactors in DNA cleavage. BLM and $\mathrm{Fe}(\mathrm{II})$ ion make a chelate complex and produce a pseudo enzyme that reacts with oxygen to produce reactive oxygen species (ROS) such as superoxide and hydroxide free radicals that can cleave DNA.

The purpose of this study was to present a novel therapeutic strategy using a combination of intracellular MNPs under an AMF and BLM, and to evaluate its therapeutic effect using tumor-bearing mice. We also evaluated the usefulness of MPI for visualizing and quantitatively evaluating the temporal changes of the MNPs in tumors.

\section{Materials and Methods}

\subsection{Reagents}

Resovist $^{\oplus}$ (Ferucarbotran, carboxydextran-coated $\gamma-\mathrm{Fe}_{2} \mathrm{O}_{3} \mathrm{MNPs}$ ) was purchased 
from FUJIFILM RI Pharma Co., Ltd. (Tokyo, Japan). HVJ-E (GenomONE ${ }^{\mathrm{TM}}-\mathrm{Neo}$ ) was purchased from ISHIHARA SANGYO Co., Ltd. (Osaka, Japan). Bleomycin hydrochloride $\left(\mathrm{Bleo}^{\oplus}\right)$ was purchased from Nippon Kayaku Co., Ltd. (Tokyo, Japan).

\subsection{Cell Line}

Colon-26 (a murine colon carcinoma cell line) was obtained from Riken Bio Resource Center (Ibaraki, Japan). The cells were maintained in $10 \mathrm{~mL}$ Roswell Park Memorial Institute (RPMI) medium containing $10 \%$ fetal bovine serum (FBS) and incubated in a humidified incubator at $37^{\circ} \mathrm{C}$ filled with air containing $5 \%$ carbon dioxide. This cell line was used at a sub-confluent state and for no more than 20 passages.

\subsection{Preparation of HVJ-E/MNPs}

MNPs were incorporated into HVJ-E (HVJ-E/MNPs) as illustrated in Figure 1 [22]. First, one assay unit (AU) of the HVJ-E vector (GenomONE $\left.{ }^{\mathrm{TM}}-\mathrm{Neo}\right)(40$ $\mu \mathrm{L}, 1 \times 10^{9}$ particles) was centrifuged at $10,000 \times \mathrm{g}$ for $5 \mathrm{~min}$ at $4^{\circ} \mathrm{C}$. The supernatant was removed and the HVJ-E pellet was suspended in phosphate buffered saline (PBS). Second, MNPs (Resovist ${ }^{\circledR}$ ) with a volume of $7.5 \mu \mathrm{L}$ (209 $\mu \mathrm{g}$ iron) and $2-\mu \mathrm{L}$ reagent $\mathrm{B}$ (polyoxyethyleneoctyl phenyl ether, Triton $\mathrm{X}-100$ ) were added and gently pipetted. Third, the suspended solution was centrifuged again at $10,000 \times \mathrm{g}$ for $5 \mathrm{~min}$ at $4^{\circ} \mathrm{C}$ to incorporate MNPs into HVJ-E (HVJ-E/MNPs), and the supernatant was removed. Finally, the HVJ-E/MNPs pellet was washed with PBS to remove both the reagent $\mathrm{B}$ and unincorporated MNPs, and was suspended in PBS, adjusting the total volume to $100 \mu \mathrm{L}$. This sample was used in subsequent experiments.

The amount of iron encapsulated into HVJ-E was quantified by colorimetry using the thiocyanate method [23]. A $100 \mu \mathrm{L}$ sample obtained by the procedure described above (Figure 1) was dissolved in $900 \mu \mathrm{L}$ of 5 -M hydrochloric acid and $120 \mu \mathrm{L}$ of $5-\mathrm{M}$ potassium thiocyanate solution was added. The colored solution $(100 \mu \mathrm{L})$ was dispensed onto each well of a 96-well microplate (Iwaki \& Co., Ltd., Tokyo, Japan), and the absorption at $480 \mathrm{~nm}$ was measured using an absorption photometer (xMark ${ }^{\mathrm{TM}}$ Microplate Spectrophotometer, Bio-Rad Laboratories, Inc., Tokyo, Japan). The amount of iron in the sample was calculated from the standard curve between the absorbance and amount of iron, measured in advance. The encapsulation efficiency was calculated by dividing the amount of iron encapsulated into HVJ-E by that of the added MNPs.

\subsection{Animal Experiments}

\subsubsection{Tumor-Bearing Mice}

All animal experiments were approved by the animal ethics committee at Osaka University School of Medicine.

Seven-week-old male BALB/c mice weighing $27.5 \pm 1.2 \mathrm{~g}$ (mean \pm standard deviation) were purchased from Charles River Laboratories Japan, Inc. (Kanagawa, 


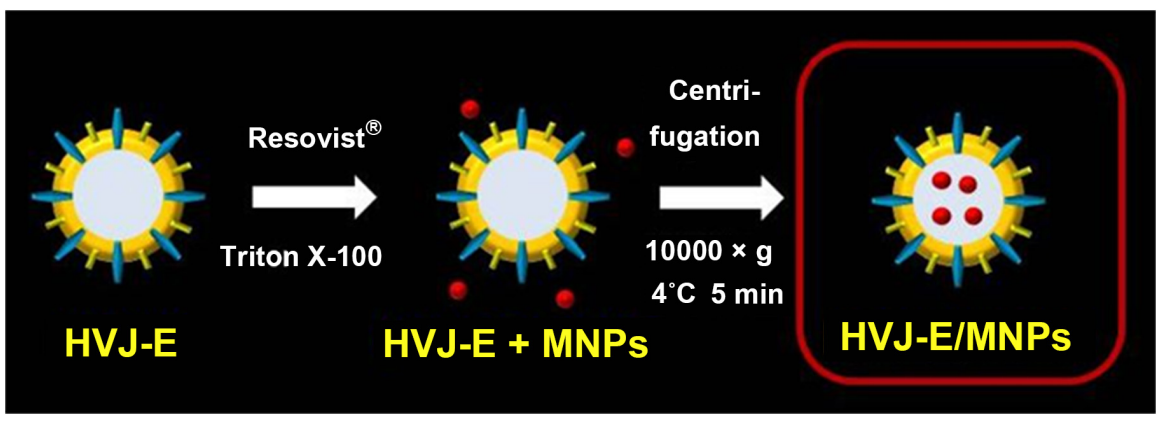

Figure 1. Illustration of a procedure for incorporating magnetic nanoparticles (MNPs) (Resovist $^{\varpi}$ ) into the hemagglutinating virus of Japan-envelope (HVJ-E) vector (HVJ-E/MNPs). The MNPs are incorporated into the HVJ-E vector by centrifugation at $10000 \times \mathrm{g}$ for $5 \mathrm{~min}$ at $4^{\circ} \mathrm{C}$ after adding Resovist ${ }^{\oplus}$ and Triton X-100 (polyoxyethylene octyl phenyl ether) to the HVJ-E vector kit (GenomONE $\left.{ }^{\mathrm{TM}}-\mathrm{Neo}\right)$.

Japan), and were housed for seven days before experiments for habituation to the rearing environment. The animals were allowed free access to food and water, and were kept under standard laboratory conditions of $22^{\circ} \mathrm{C}-23^{\circ} \mathrm{C}$ room temperature, around 50\% humidity, and a 12:12 hour light/dark cycle.

After Colon-26 cells were enzymatically detached from a culture dish and the number of cells was counted, the cells $\left(1 \times 10^{6}\right.$ cells $)$ were suspended in $100 \mu \mathrm{L}$ of PBS and implanted subcutaneously into the back of each mouse under pentobarbital sodium anesthesia (Somnopentyl $1^{\oplus}$, Kyoritsu Seiyaku Co., Ltd., Tokyo, Japan) $(0.001 \mathrm{~mL} / \mathrm{g}$ body weight). Tumors started to appear at 7 to 10 days after the inoculation.

\subsubsection{AMF Treatment}

When the tumor volume reached approximately $100 \mathrm{~mm}^{3}, 5$ AUs of HVJ-E/MNPs and/or BLM $(20 \mu \mathrm{g})$ were directly injected into the tumor. After the injection of agents, the syringe needle was retracted slowly to avoid leakage. The total injected volume was fixed at $100 \mu \mathrm{L}$ in all cases.

One hour after the injection of HVJ-E/MNPs or both BLM and HVJ-E/MNPs, each mouse was placed in a holder and set in the coil for generating an AMF [24]. This coil consists of 19-turned loops $(6.5 \mathrm{~cm}$ in diameter and $10 \mathrm{~cm}$ in length) of copper pipe (5 $\mathrm{mm}$ in diameter) and is cooled with water to ensure constant temperature and impedance. The coil was connected to a high-frequency power supply (T162-5723BHE, Thamway Co., Ltd., Shizuoka, Japan) and a manual-matching unit (T020-5723AHE, Thamway Co., Ltd., Shizuoka, Japan). The amplitude of the AMF generated in the coil can be controlled by changing the output of the power supply. In this study, the frequency and peak amplitude of the AMF were fixed at $600 \mathrm{kHz}$ and $3.1 \mathrm{kA} / \mathrm{m}$, respectively, and the AMF was applied to the above mice for $20 \mathrm{~min}$ [9] [18]. Note that we refer to the treatment using HVJ-E/MNPs and AMF as the AMF treatment. Neither BLM nor AMF treatment was carried out on the mice in the control group.

During the AMF treatment, the temperatures in the tumor and rectum were 
measured in four mice using two fluorescence-type optical fiber thermometers (AMOTH FL-2000, Anritsu Meter Co., Ltd., Tokyo, Japan) and two optical fiber temperature probes. One probe was inserted into the center of the tumor and the other probe was inserted $1 \mathrm{~cm}$ inside the rectum. Both temperatures were recorded every minute until the end of the AMF treatment.

\subsubsection{Magnetic Particle Imaging}

MPI was performed using our MPI scanner, a photograph of which is shown in Figure 2. In our MPI scanner, a field-free-line (FFL) encoding scheme was adopted and the selection magnetic field was generated by two opposing neodymium magnets [16] [19]. The gradient strengths of the selection magnetic field perpendicular and parallel to the FFL were $3.9 \mathrm{~T} / \mathrm{m}$ and $0.1 \mathrm{~T} / \mathrm{m}$, respectively. An excitation coil for generating a drive magnetic field and a gradiometer-type receiving coil were placed between the two neodymium magnets. The frequency and peak-to-peak amplitude of the drive magnetic field were taken as $400 \mathrm{~Hz}$ and $20 \mathrm{mT}$, respectively [16] [19]. The third-harmonic component was extracted from the signal received by the gradiometer coil using a lock-in amplifier (LI5640, NF Co., Ltd., Yokohama, Japan). The output of the lock-in amplifier was converted to digital data by a personal computer connected to a multifunction data acquisition device (USB-6212, National Instruments Co., Ltd., TX, USA).

Figure 3(a) illustrates the protocol for data acquisition in the AMF group in which HVJ-E/MNPs was injected directly into the tumor, while Figure 3(b) illustrates that in the BLM + AMF group in which both BLM and HVJ-E/MNPs were injected directly into the tumor. As illustrated in Figure 3, the tumor-bearing mice injected with HVJ-E/MNPs were scanned using our MPI scanner immediately (13 $\mathrm{min}$ ) before, immediately ( $22 \mathrm{~min}$ ) after, and 3, 7, and 14 days after AMF treatment. For MPI scans, the anesthetized mouse was placed in the receiving coil and its position adjusted at the maximum MPI signal intensity to determine the imaging slice. In order to acquire projection data for image reconstruction, the mouse in the receiving coil was automatically rotated around the axis of the receiving coil through $180^{\circ}$ in steps of $5^{\circ}$ and translated in the direction perpendicular to the axis of the receiving coil from $-16 \mathrm{~mm}$ to 16 $\mathrm{mm}$ in steps of $1 \mathrm{~mm}$, using an XYZ-axes rotary stage (HPS80-50X-M5, Sigma Koki Co., Ltd., Tokyo, Japan). The rotation and translation were controlled using LabVIEW (National Instruments Co., Ltd., TX, USA). Data acquisition took about $12 \mathrm{~min}$. Each projection data set was then transformed into 64 bins by linear interpolation. After the inhomogeneous sensitivity of the receiving coil and feedthrough interference were corrected [25], transverse images were reconstructed from the projection data using the maximum likelihood-expectation maximization (ML-EM) algorithm with an iteration number of 15 [16] [17].

After the MPI scans, X-ray CT images were obtained using a 4-row multi-slice CT scanner (Asteion, Toshiba Medical Systems Co., Ltd., Tokyo, Japan) with a 


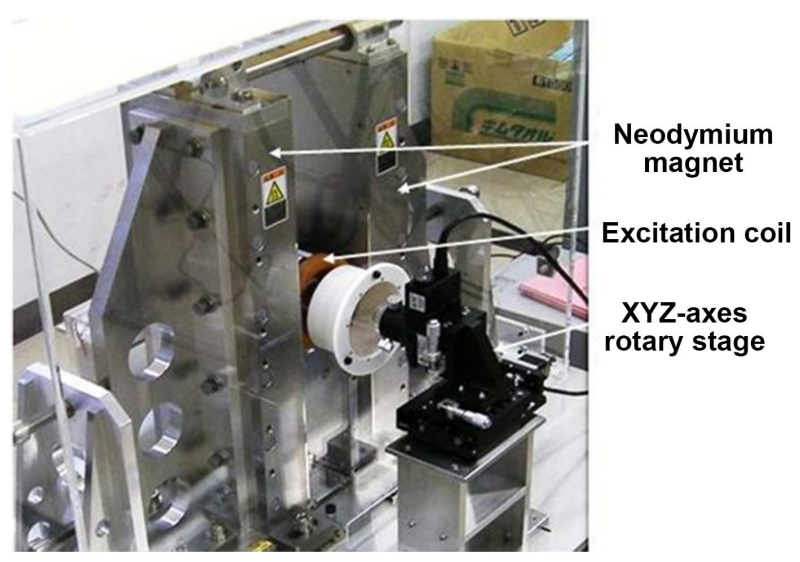

Figure 2. Photograph of our scanner for magnetic particle imaging (MPI). In our MPI scanner, a field-free-line (FFL) encoding scheme is used and a selection magnetic field is generated by two opposing neodymium magnets. An excitation coil for generating a drive magnetic field and a gradiometer-type receiving coil are placed between the two neodymium magnets.

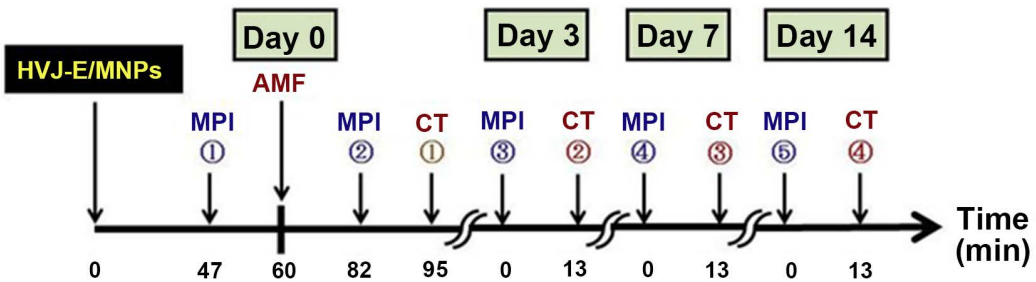

(a)
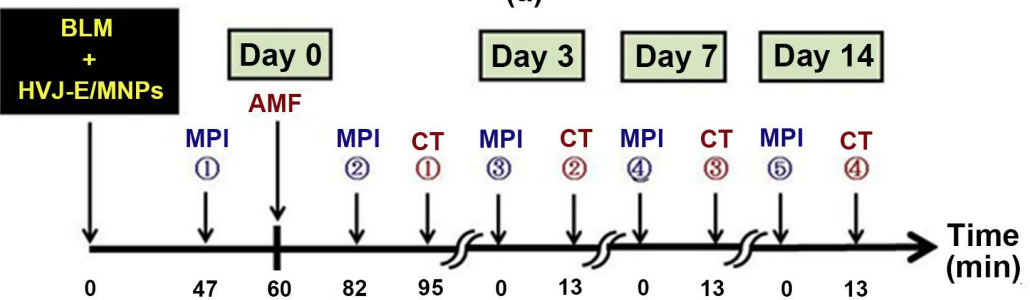

(b)

Figure 3. Protocol for data acquisition: (a) for the AMF group in which HVJ-E/MNPs were injected directly into the tumor and (b) for the BLM + AMF group in which both bleomycin (BLM) and HVJ-E/MNPs were injected directly into the tumor. AMF: alternating magnetic field, MPI: magnetic particle imaging, and CT: X-ray computed tomography.

tube voltage of $120 \mathrm{kV}$, a tube current of $210 \mathrm{~mA}$, and a slice thickness of 0.5 $\mathrm{mm}$ (Figure 3). The X-ray CT image obtained after the second MPI study was substituted for that after the first MPI study. The MPI images were co-registered to the X-ray CT images for anatomical identification using the method reported in our previous paper [18].

\subsection{Transmission Electron Microscopic Study}

Transmission electron microscopic (TEM) studies were performed to investigate the intracellular distributions of MNPs in the tumors at the time of AMF 
treatment. Separately from the animal experiments described above, seven-week-old male BALB/c mice were purchased for the TEM studies and were implanted with Colon-26 cells in the same manner as described above. When the tumor volume reached approximately $100 \mathrm{~mm}^{3}$, the tumor was injected with HVJ-E/MNPs or only MNPs. The amount of iron and total injected volume were taken to be the same as described above. The mice were sacrificed one hour after the injection of agents, and the tumors were resected. The resected tumor tissues were fixed in mixture of $2 \%$ paraformaldehyde and $2.5 \%$ glutaraldehyde in $0.1 \%$ PBS (Wako Pure Chemical Industries, Ltd., Osaka, Japan) at $4^{\circ} \mathrm{C}$. The microstructures of these tumor tissues were observed using an electron microscope (JEM-1011, JEOL Co., Ltd., Tokyo, Japan).

\subsection{Data Analysis}

The vertical diameter $\left(L_{v d}\right)$, horizontal diameter $\left(L_{h d}\right)$, and height $\left(L_{h}\right)$ of the tumor were measured with a caliper every day until 14 days after AMF treatment. The tumor volume $(V)$ was calculated using the following formula:

$$
V=\frac{\pi}{6} \times L_{v d} \times L_{h d} \times L_{h}
$$

The relative tumor volume growth (RTVG) was calculated from

$$
R T V G=\frac{V-V_{0}}{V_{0}}
$$

In the control group, $V_{0}$ was taken as the tumor volume immediately after it exceeded $100 \mathrm{~mm}^{3}$. In the AMF and BLM + AMF groups, $V_{0}$ was taken as the tumor volume immediately before the AMF treatment. In the BLM group, $V_{0}$ was taken as the tumor volume immediately before the injection of BLM. In this study, the RTVG value was used as an index of the therapeutic effect.

To quantitatively evaluate the time-dependent change of the MNPs in the tumor, we drew a region of interest (ROI) on the tumor in the MPI image and calculated the average and maximum MPI values within the ROI. The threshold value for extracting the contour of the tumor was taken as $40 \%$ of the maximum MPI value within the ROI. Note that we refer to the pixel value of the MPI image as the MPI value in this study [18].

\subsection{Statistical Analysis}

In animal experiments, one-way analysis of variance (ANOVA) was used for comparison of the RTVG values among groups. As a post-hoc test, the Tukey-Kramer multiple comparison test was used to determine significance. The Student's $t$-test was used for comparisons of the average and maximum MPI values between groups at each time point and between time points in each group. A $p$ value less than 0.05 was considered significant.

\section{Results}

The amount of iron encapsulated into HVJ-E per one AU was $88.3 \pm 3.6 \mu \mathrm{g}$ 
[mean \pm standard error $(\mathrm{SE})$ for $\mathrm{n}=3$ ]. This means that approximately $442 \mu \mathrm{g}$ iron was injected into the tumor in animal experiments, because 5 AUs of HVJ-E/MNPs were injected into the tumor. The encapsulation efficiency was $42.2 \% \pm 1.7 \%$ (mean \pm SE for $\mathrm{n}=3$ ).

Figure 4 shows the time courses of the temperatures in the tumor and rectum in the AMF group $(n=4)$. Although the mean temperature in the tumor rose by approximately $1.3^{\circ} \mathrm{C}$ and that in the rectum decreased by approximately $1.9^{\circ} \mathrm{C}$ during the AMF treatment, there were no significant differences between the temperatures at the beginning and end of the AMF treatment in both the tumor and rectum.

Figure 5 shows the time courses of the RTVG values calculated from Equation (2) in the control $(\mathrm{n}=9)$, AMF $(\mathrm{n}=10), \operatorname{BLM}(\mathrm{n}=11)$, and BLM + AMF groups $(\mathrm{n}=8)$. The RTVG value in the BLM + AMF group was significantly lower than that in the control group one day or more after AMF treatment (shown by ${ }^{*}$ ), and was significantly lower than that in the AMF group at 9 to 14 days after AMF treatment (shown by \#). It was also significantly lower than that in the BLM group at 3 to 5 days after AMF treatment (shown by $\dagger$ ). There were significant differences in the RTVG values between the control and AMF groups at 2 to 6 days (shown by \$), and between the control and BLM groups at 3 to 11 days after AMF treatment (shown by \&).

Figure 6 shows the MPI images superimposed on the X-ray CT images immediately ( $13 \mathrm{~min}$ ) before, immediately ( $22 \mathrm{~min}$ ) after, and 3, 7, and 14 days after AMF treatment. The upper and lower rows show those in the AMF and BLM + AMF groups, respectively. As shown in Figure 6, the MPI values in the tumors did not change largely with time in both groups.

Figure 7(a) and Figure 7(b) show the temporal changes of the average and maximum MPI values, respectively, in the AMF (red bars) and BLM + AMF groups (blue bars). There were no significant differences in the average and maximum MPI values between the two groups. There were also no significant temporal changes in either the average or maximum MPI values.

Figure 8(a) and Figure 8(b) show the TEM images of the resected tumors one hour after the injection of HVJ-E/MNPs and only MNPs, respectively. The TEM images showed most of the MNPs to be present in the cytoplasm when HVJ-E/MNPs were injected [Figure 7(a)], while most of the MNPs remained in the extracellular space when only MNPs were injected [Figure 7(b)].

\section{Discussion}

In this study, we devised a new therapeutic strategy using intracellular MNPs under an AMF (AMF treatment) combined with an anticancer antibiotic, BLM, and investigated its therapeutic effect as compared with that of the AMF treatment or BLM alone. We used HVJ-E to enhance the internalization of MNPs into tumor cells. We also used MPI to quantitatively investigate the time-dependent change of the distribution of MNPs in the tumor. The present 


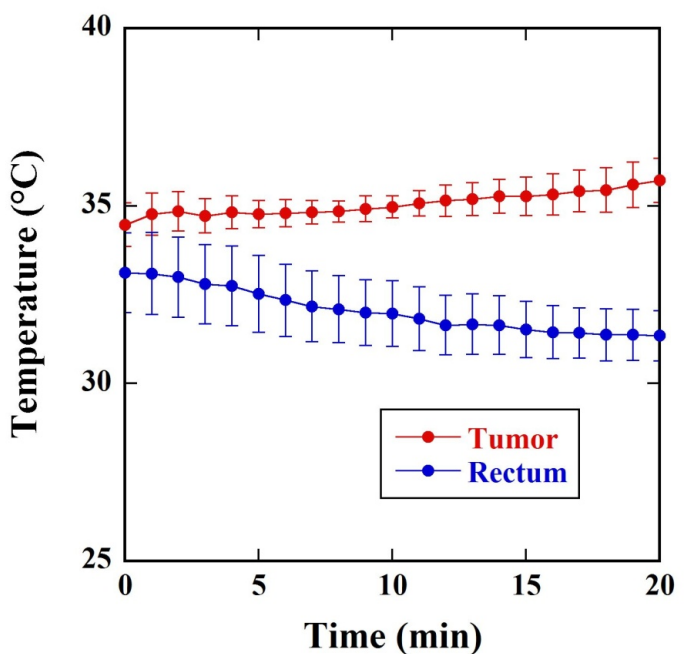

Figure 4. Temporal changes of the temperature in the tumor (red circles) and rectum (blue circles) during AMF treatment. The AMF treatment was performed for $20 \mathrm{~min}$, starting one hour after the injection of HVJ-E/MNPs. Data are represented as the mean \pm standard error (SE) for $n=4$.

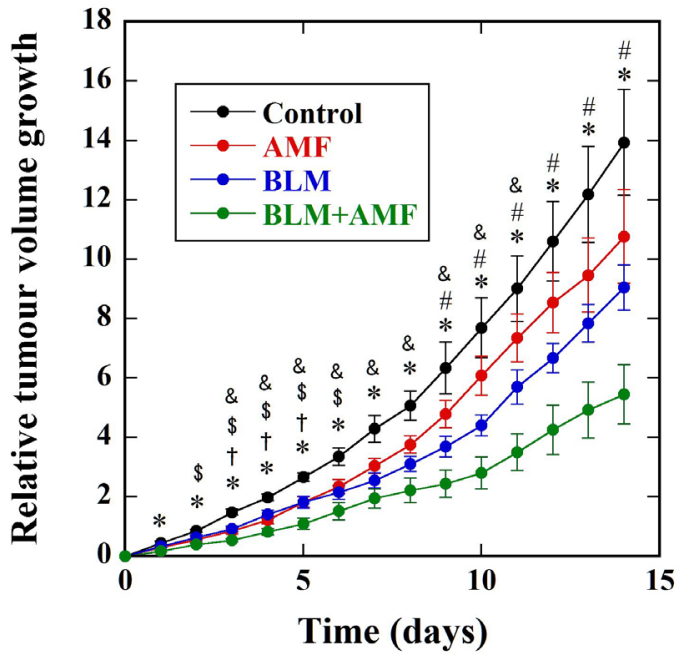

Figure 5. Time courses of the relative tumor volume growth (RTVG) values in the control (black circles, $\mathrm{n}=10$ ), AMF (red circles, $\mathrm{n}=10$ ), BLM (blue circles, $\mathrm{n}=11$ ), and BLM + AMF groups (green circles, $\mathrm{n}=8$ ). In the control group, neither BLM nor AMF treatment was carried out. In the BLM group, BLM was injected directly into the tumor and the AMF treatment was not performed. *: $p<0.05$ between the control and BLM + AMF groups. \#: $p<$ 0.05 between the AMF and BLM + AMF groups. $\dagger: p<0.05$ between the BLM and BLM + AMF groups. $\$: p<0.05$ between the AMF and control groups. \&: $p<0.05$ between the BLM and control groups. Data are represented as the mean \pm SE.

results (Figures 4-8) suggested that the AMF treatment combined with BLM is effective for cancer therapy and that MPI is useful for the in vivo longitudinal observation of MNPs in tumors. 


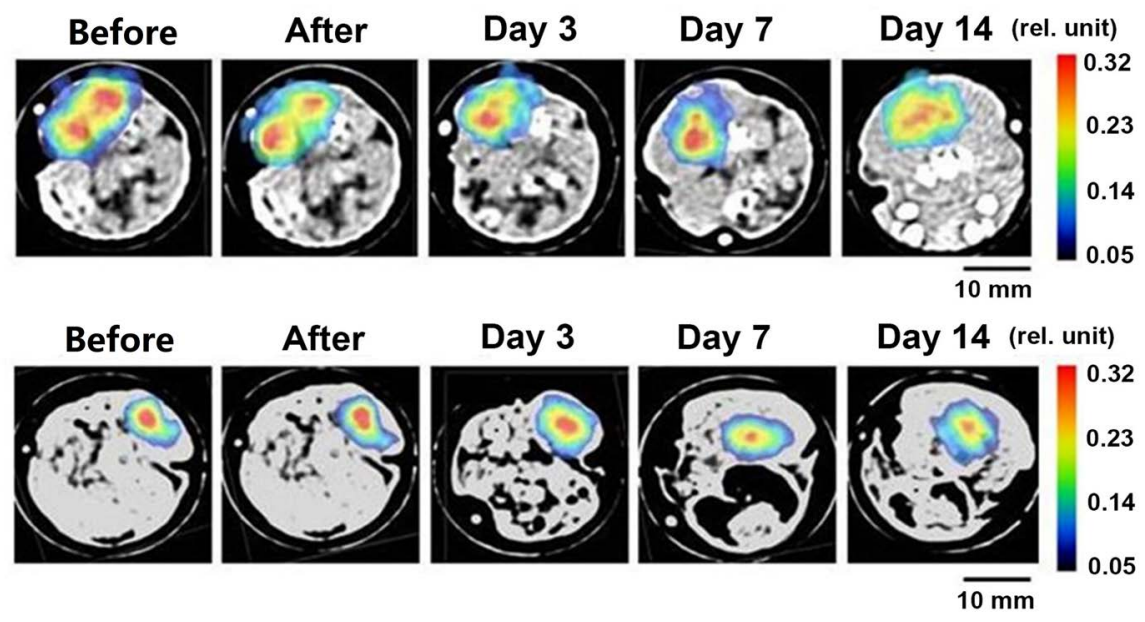

Figure 6. MPI images superimposed on the X-ray CT images immediately (13 $\mathrm{min}$ ) before, immediately (22 $\mathrm{min}$ ) after, and 3, 7, and 14 days after AMF treatment. The upper and lower rows show images from the AMF and BLM + AMF groups, respectively. Scale bar $=10 \mathrm{~mm}$.

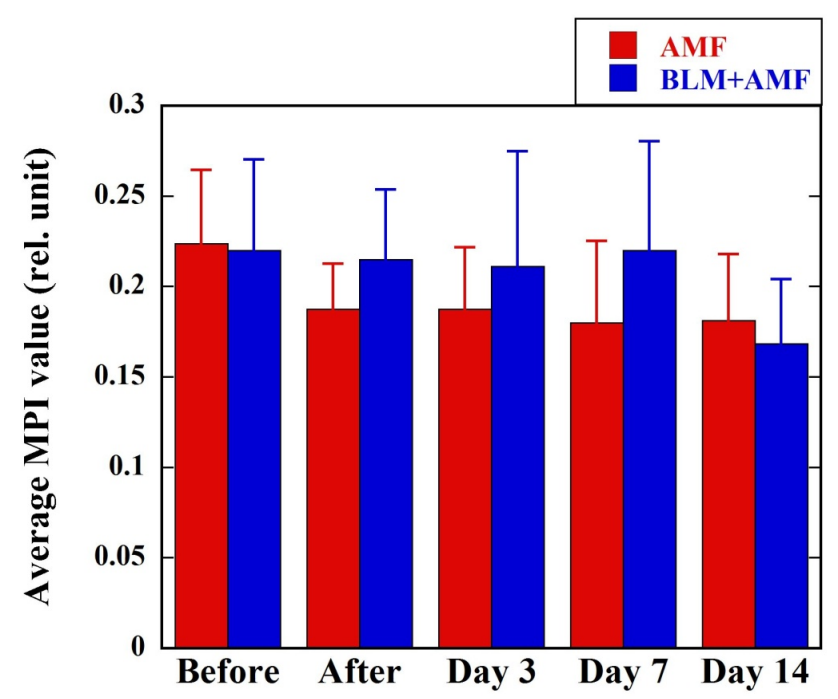

(a)

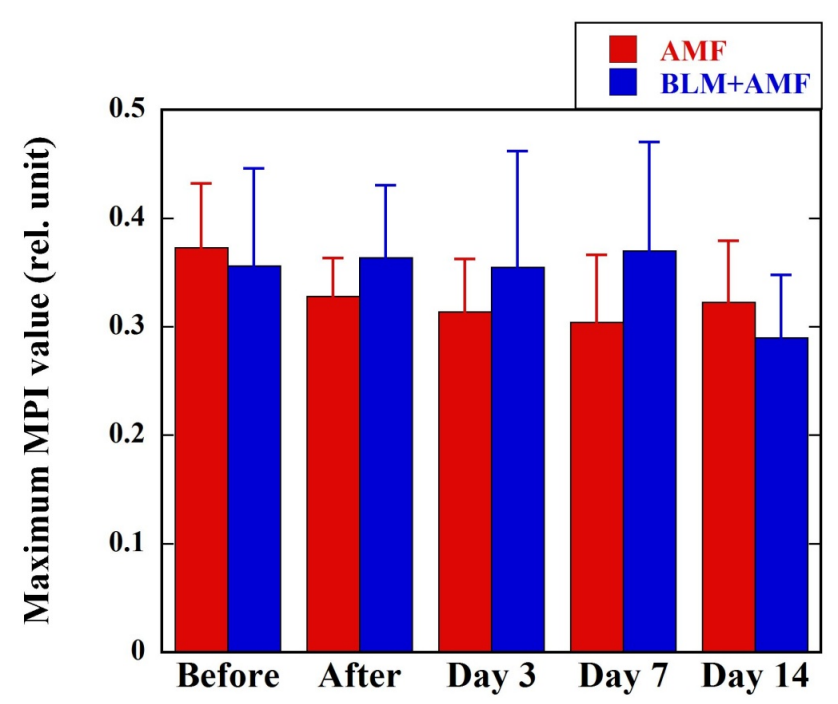

(b)

Figure 7. Temporal changes of the average (a) and maximum MPI values (b) immediately (13 min) before, immediately (22 min) after, and 3, 7, and 14 days after AMF treatment. The red and blue bars indicate the AMF $(\mathrm{n}=10)$ and BLM + AMF groups $(\mathrm{n}=$ 8), respectively. The error bar represents SE.

As previously described, MNPs (Resovist ${ }^{\oplus}$ ) with a volume of $7.5 \mu \mathrm{L}(209 \mu \mathrm{g}$ iron) were used per one AU of HVJ-E. In this case, the amount of iron encapsulated into HVJ-E and the encapsulation efficiency were approximately $88 \mu \mathrm{g}$ and $42 \%$, respectively. Although it was possible to increase the amount of iron encapsulated into HVJ-E by increasing the volume of MNPs added, the amount of encapsulated iron and encapsulation efficiency gradually saturated and decreased, respectively, with increasing volume of MNPs added (data not shown). Thus, we adopted $7.5 \mu \mathrm{L}$ as the volume of MNPs (Resovist ${ }^{\circledast}$ ) added to one AU of HVJ-E, to minimize the loss of MNPs. 


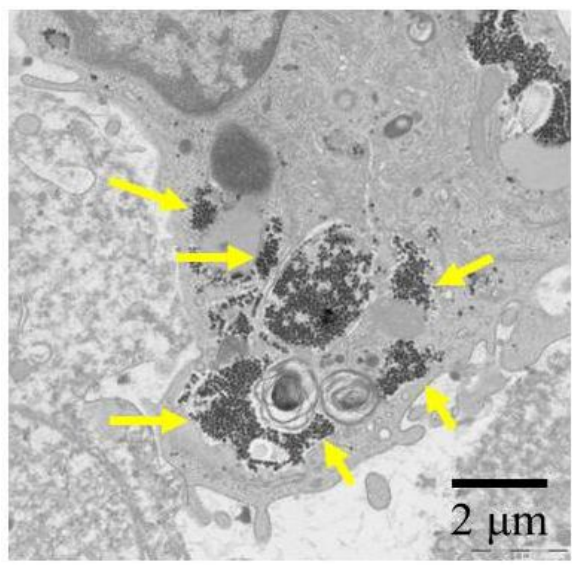

(a)

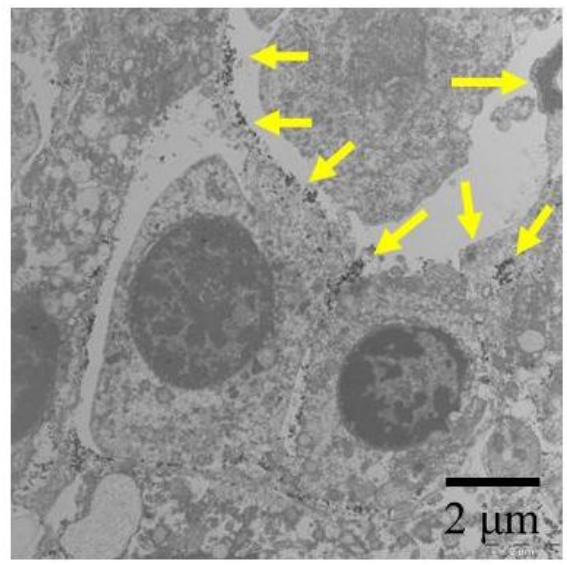

(b)

Figure 8. Transmission electron microscopic (TEM) images of resected tumor tissues one hour after the injection of HVJ-E/MNPs (a) and only MNPs (b). Arrows indicate MNPs. Scale bar $=2 \mu \mathrm{m}$.

As previously described, we measured the temperature of the tumor during AMF treatment using a fluorescence-type optical fiber thermometer, and found that it did not significantly increase from the temperature at the start of the AMF treatment (approximately $34^{\circ} \mathrm{C}$ ) (Figure 4). Because it is generally known that hyperthermia treatment requires a temperature over approximately $42.5^{\circ} \mathrm{C}$ to damage cancer cells, we initially thought that the AMF treatment had no therapeutic effect. As shown in Figure 5, however, the RTVG value in the AMF group was significantly lower than that in the control group at 2 to 6 days after the treatment, implying that the AMF treatment can damage cancer cells without a notable temperature rise (Figure 4). Wydra et al. reported that, with exposure to an AMF, MNPs cause a significant increase in cellular ROS and apoptotic cell death with no measurable increase in temperature in vivo [26]. They presumed that this is caused by the mechanical motion of the MNPs gathered in lysosomes; the MNPs rotate physically to realign themselves with the magnetic field in the presence of the AMF, and these physical motions of MNPs induce stress within the cells and increase ROS generation [26]. Other reports suggested that, during intracellular magnetic hyperthermia, the mechanical vibration of MNPs generates highly localized heat around the MNPs without a bulk temperature rise, which would damage the cells from the inside and induce apoptosis [27]. The present results (Figure 5) appear to be related to these phenomena of ROS generation and highly localized heat due to the mechanical motion and vibration of MNPs under the AMF. The fact that the AMF treatment had a therapeutic effect without a significant temperature rise appears to be advantageous, because it does not induce heat-shock protein. In conventional hyperthermia with temperatures above $42.5^{\circ} \mathrm{C}$, a significant amount of heat-shock protein is expressed within and/or around the tumor tissues, resulting in the generation of tumor hyperthermia resistance [1]. AMF treatment without a significant temperature rise might permit repeated treatment without generating any hyperthermia resistance. 
The combination of the AMF treatment and BLM resulted in significant tumor growth suppression compared to AMF treatment or BLM alone, because the RTVG value in the BLM + AMF group was significantly lower than that in the AMF group at 9 to 14 days and that in the BLM group at 3 to 5 days after the treatment (Figure 5). This may be due to the fact that the combination of the AMF treatment and chemotherapy with BLM synergistically increased ROS generation, and the ROS production exceeded the repair capacity of cancer cells.

BLM is a highly hydrophilic glycoprotein, and no BLM-specific receptors are expressed on cell membranes [28]. The sensitivity of tumor cells to BLM is highly variable due to the limited penetration of BLM through the plasma membrane [28]. As in the case of macromolecules, BLM is practically non-permeant to the plasma membrane and enters the cells by endocytosis, whereby it may be either degraded in the lysosomes, or due to the slow entrance into the cytosol, degraded by BLM hydrolase before reaching its therapeutic target in the nucleus [29] [30]. The use of HVJ-E appears to be a powerful tool for overcoming such problems; incorporating BLM into HVJ-E would enable much easier penetration through the plasma membrane and prevent BLM degradation by hydrolytic enzymes in late endosomes. Mima et al. reported that BLM could be delivered to cancer cells by the HVJ-E vector approximately 300-fold more effectively than by BLM alone [31]. Additionally, it has been reported that a combination therapy of BLM and cis-diammine-dichloroplatinum (II) (cisplatin, CDDP), having different working mechanisms, demonstrated a higher treatment effect than BLM or CDDP alone [32]. Therefore, BLM has the potential to be a very efficient and specific chemotherapeutic agent, by combining it with a treatment method that activates its therapeutic potential only in the target tissue, as seen in this study (Figure 5).

It has been reported that the cores of MNPs themselves show cytotoxicity, which is induced by the production of highly reactive hydroxyl radicals under conditions of the biologically relevant Fenton-like reaction with endogenous hydrogen peroxide [33]. Voinov et al. showed that this free radical production is attributed primarily to catalytic reactions at the surface of MNPs rather than being caused by dissolved metal ions released by the MNPs [33]. As described above, a combination of AMF treatment and BLM appears to synergistically increase ROS generation. The use or development of iron oxide nanoparticles that efficiently and selectively induce free radical production in cancer cells as a source for AMF treatment might further enhance the therapeutic effect of our strategy.

As shown in Figure 7, there were no significant differences in the average and maximum MPI values between the AMF and BLM + AMF groups. Furthermore, the MPI values were almost constant until day 14 in both groups (Figure 6 and Figure 7). This appears to be the main reason why MNPs were rapidly taken up by tumor cells after the injection of HVJ-E/MNPs into tumors. This feature will be especially useful when considering repeated applications of AMF treatment. The results shown in Figure 6 and Figure 7 also appear to suggest that MPI is 
useful for visualizing and quantitatively evaluating the temporal change of the distribution of MNPs.

To investigate whether HVJ-E/MNPs are internalized into tumor cells within one hour after the injection, we performed TEM studies (Figure 8). The TEM image in Figure 8(a) demonstrates that most of the HVJ-E/MNPs introduced into cells were located in intracellular spaces. We performed AMF treatment one hour after the injection of HVJ-E/MNPs (Figure 3), and it appears from this TEM image that most of the HVJ-E/MNPs were located in intracellular spaces at the time of the AMF treatment. This also appears to support the results described above (Figure 6 and Figure 7). In contrast, when MNPs were not incorporated into any vectors such as HVJ-E, they remained in extracellular spaces as shown in Figure 8(b). These results appear to indicate that HVJ-E is effective for internalizing MNPs into cancer cells. It has been reported that the cellular engulfment of MNPs via endocytosis-dependent uptake takes about six hours [34]. Therefore, the results shown in Figure 8 appear to demonstrate that the use of HVJ-E as a vector promotes quick cellular uptake. HVJ-E has two kinds of membrane protein [hemagglutinin-neuraminidase $(\mathrm{HN})$ protein and fusion $(\mathrm{F})$ protein] on its surface to infect living bodies [11]. The HN protein attaches to cells by binding with sialic acid receptors expressed on cellular surfaces. Then, the $\mathrm{HN}$ protein interacts with the $\mathrm{F}$ protein to induce efficient cell membrane fusion [35]. These mechanisms derived from viral infection appear to promote the rapid introduction of HVJ-E/MNPs into tumor cells (Figure 8). When we consider the practical application of our therapeutic strategy, this feature will be helpful for reducing the burden on patients, because the time for MNPs to be internalized into tumor cells can be shortened and the AMF treatment can be started earlier.

\section{Conclusion}

This study investigated the usefulness of a novel therapeutic strategy combining the use of intracellular MNPs under an AMF and BLM in tumor-bearing mice, and quantitatively evaluated the temporal changes of MNPs in tumors using MPI. This study also investigated the usefulness of HVJ-E for internalizing MNPs into cancer cells. Our results suggest that this novel strategy is effective for cancer therapy, because it can suppress tumor growth better than AMF treatment or BLM alone. They also suggest that this strategy can be performed repeatedly with a single injection of HVJ-E/MNPs and without generating hyperthermia resistance, because the spatial distribution of HVJ-E/MNPs in the tumor is almost constant until day 14 and the temperature of the tumor does not significantly increase during AMF treatment. In addition, our results suggest that HVJ-E is effective for internalizing MNPs into cancer cells and that MPI allows for in vivo longitudinal monitoring of the distribution of MNPs in tumors.

\section{Acknowledgements}

This work was supported by Grants-in-Aid for Scientific Research (Grant Num- 
ber: 25282131 and 15K12508) from the Japan Society for the Promotion of Science (JSPS) and the Japan Science and Technology Agency (JST).

\section{Conflicts of Interest}

The authors report no conflicts of interest.

\section{References}

[1] Mallory, M., Gogineni, E., Jones, G.C., Greer, L. and Simone, C.B. (2016) Therapeutic Hyperthermia: The Old, the New, and the Upcoming. Critical Reviews in Oncology/ Hematology, 97, 56-64. https://doi.org/10.1016/j.critrevonc.2015.08.003

[2] Murase, K., Oonoki, J., Takata, H., Song, R., Angraini, A., Ausanai, P. and Matsushita, T. (2011) Simulation and Experimental Studies on Magnetic Hyperthermia with Use of Superparamagnetic Iron Oxide Nanoparticles. Radiological Physics and Technology, 4, 194-202. https://doi.org/10.1007/s12194-011-0123-4

[3] Petryk, A.A., Giustini, A.J., Gottesman, R.E., Trembly, B.S. and Hoopes, P.J. (2013) Comparison of Magnetic Nanoparticle and Microwave Hyperthermia Cancer Treatment Methodology and Treatment Effect in a Rodent Breast Cancer. International Journal of Hyperthermia, 29, 819-827.

https://doi.org/10.3109/02656736.2013.845801

[4] Mahmoudi, M., Sant, S., Wang, B., Laurent, S. and Sen, T. (2011) Superparamagnetic Iron Oxide Nanoparticles (SPIONs): Development, Surface Modification and Applications in Chemotherapy. Advanced Drug Delivery Reviews, 63, 24-46. https://doi.org/10.1016/j.addr.2010.05.006

[5] Polyak, B., Fishbein, I., Chorny, M., Alferiev, I., Williams, D., Yellen, B., Friedman, G. and Levy, R.J. (2008) High Field Gradient Targeting of Magnetic Nanoparticle-Loaded Endothelial Cells to the Surfaces of Steel Stents. Proceedings of the National Academy of Sciences of the United States of America, 105, 698-703. https://doi.org/10.1073/pnas.0708338105

[6] Hoopes, P.J., Petryk, A.A., Tate, J.A., Savellano, M.S., Strawbrige, R.R., Giustini, A.J., Stan, R.V., Gimi, B. and Garwood, M. (2013) Imaging and Modification of the Tumor Vascular Barrier for Improvement in Magnetic Nanoparticle Uptake and Hyperthermia Treatment Efficacy. Proceedings of SPIE, 8584, Article ID: 8584403. https://doi.org/10.1117/12.2008689

[7] Gordon, R.T., Hines, J.R. and Gordon, D. (1979) Intracellular Hyperthermia a Biophysical Approach to Cancer Treatment via Intracellular Temperature and Biophysical Alterations. Medical Hypotheses, 5, 83-102. https://doi.org/10.1016/0306-9877(79)90063-X

[8] Jordan, A., Wust, P., Fahling, H., John, W., Hinz, A. and Felix, R. (1993) Inductive Heating of Ferromagnetic Particles and Magnetic Fluids: Physical Evaluation of Their Potential for Hyperthermia. International Journal of Hyperthermia, 9, 51-68. https://doi.org/10.3109/02656739309061478

[9] Kobayashi, S., Ohki, A., Tanoue, M., Inaoka, Y. and Murase, K. (2017) Comparative Study of Extracellular and Intracellular Magnetic Hyperthermia Treatments Using Magnetic Particle Imaging. Open Journal of Applied Sciences, 7, 647-660. https://doi.org/10.4236/ojapps.2017.712047

[10] Thu, S., Bryant, L.H., Coppola, T., Jordan, E.K., Budde, M.D., Lewis, B.K., Ren, J., Varma, N.R., Arbab, A.S. and Frank, J.A. (2012) Self-Assembling Nanocomplexes by Combining Ferumoxytol, Heparin and Protamine for Cell Tracking by Magnetic Resonance Imaging. Nature Medicine, 18, 463-467. 
https://doi.org/10.1038/nm.2666

[11] Kaneda, Y., Nakajima, T., Nishikawa, T., Yamamoto, S., Ikegami, H., Suzuki, N., Nakamura, H., Morishita, R. and Kotani, H. (2002) Hemagglutinating Virus of Japan (HVJ) Envelope Vector as a Versatile Gene Delivery System. Molecular Therapy, 6, 219-226. https://doi.org/10.1006/mthe.2002.0647

[12] Kurooka, M. and Kaneda, Y. (2007) Inactivated Sendai Virus Particles Eradicate Tumors by Inducing Immune Responses through Blocking Regulatory $\mathrm{T}$ Cells. Cancer Research, 67, 227-236. https://doi.org/10.1158/0008-5472.CAN-06-1615

[13] Inai, M., Yamauchi, M., Honda, N., Hazama, H., Tachikawa, S., Nakamura, H., Nishida, T., Yasuda, H., Kaneda, Y. and Awazu, K. (2015) Photodynamic Therapy Using Hemagglutinating Virus of Japan Envelope (HVJ-E): A Novel Therapeutic Approach for the Treatment of Hormone Antagonistic Prostate Cancer. Proceedings of SPIE, 9308, Article ID: 930814.

[14] Takahashi, Y., Tomotsune, D., Takizawa, S., Yue, F., Nagai, M., Yokoyama, T., Hirashima, K. and Sasaki, K. (2015) New Model for Cardiomyocyte Sheet Transplantation Using a Virus-Cell Fusion Technique. World Journal of Stem Cells, 7, 883-893. https://doi.org/10.4252/wjsc.v7.i5.883

[15] Gleich, B. and Weizenecker, J. (2005) Tomographic Imaging Using the Nonlinear Response of Magnetic Particles. Nature, 435, 1214-1217. https://doi.org/10.1038/nature03808

[16] Murase, K., Hiratsuka, S., Song, R. and Takeuchi, Y. (2014) Development of a System for Magnetic Particle Imaging Using Neodymium Magnets and Gradiometer. Japanese Journal of Applied Physics, 53, Article ID: 067001. https://doi.org/10.7567/JAP.53.067001

[17] Murase, K., Song, R. and Hiratsuka, K. (2014) Magnetic Particle Imaging of Blood Coagulation. Applied Physics Letters, 104, Article ID: 2524409. https://doi.org/10.1063/1.4885146

[18] Murase, K., Aoki, M., Banura, N., Nishimoto, K., Mimura, A., Kuboyabu, T. and Yabata, I. (2015) Usefulness of Magnetic Particle Imaging for Predicting the Therapeutic Effect of Magnetic Hyperthermia. Open Journal of Medical Imaging, 5, 85-99. https://doi.org/10.4236/ojmi.2015.52013

[19] Umezawa, H., Maeda, K., Takeuchi, T. and Okami, Y. (1966) New Antibiotics, Bleomycin A and B. Journal of Antibiotics, 19, 200-209.

[20] Ohguri, T., Imada, H., Narisada, H. and Korogi, Y. (2007) Clinical Results of Systemic Chemotherapy Combined with Regional Hyperthermia. Thermal Medicine, 23, 49-61. https://doi.org/10.3191/thermalmedicine.23.49

[21] Burger, R.M., Peisach, J. and Horwitz, S.B. (1981) Activated Bleomycin. A Transient Complex of Drug, Iron, and Oxygen That Degrades DNA. Biological Chemistry, 256, 11636-11644.

[22] Toyoda, K., Tooyama, I., Kato, M., Sato, H., Morikawa, S., Hisa, Y. and Inubushi, T. (2004) Effective Magnetic Labeling of Transplanted Cells with HVJ-E for Magnetic Resonance Imaging. Neuroreport, 15, 589-593. https://doi.org/10.1097/00001756-200403220-00004

[23] Shamsipour, F., Zarnani, A.H., Ghods, R., Chamankhah, M., Forouzesh, F., Vafaei, S., Bayat, A.A., Akhondi, M.M., Oghabian, M.A. and Jeddi-Tehrani, M. (2009) Conjugation of Monoclonal Antibodies to Super Paramagnetic Iron Oxide Nanoparticles for Detection of Her2/Neu Antigen on Breast Cancer Cell Lines. Avicenna Journal of Medical Biotechnology, 1, 27-31. 
[24] Ohki, A., Tanoue, M., Kobayashi, S. and Murase, K. (2017) Magnetic Particle Imaging for Quantitative Evaluation of Tumor Response to Magnetic Hyperthermia Treatment Combined with Chemotherapy Using Cisplatin. Thermal Medicine, 33, 39-51. https://doi.org/10.3191/thermalmed.33.39

[25] Murase, K., Banura, N., Mimura, A. and Nishimoto, K. (2015) Simple and Practical Method for Correcting the Inhomogeneous Sensitivity of a Receiving Coil in Magnetic Particle Imaging. Japanese Journal of Applied Physics, 54, Article ID: 038001. https://doi.org/10.7567/JAP.54.038001

[26] Wydra, R.J., Rychahou, P.G., Evers, B.M., Anderson, K.W., Dziubla, T.D. and Hilt, J.Z. (2015) The Role of ROS Generation from Magnetic Nanoparticles in an Alternating Magnetic Field on Cytotoxicity. Acta Biomaterialia, 25, 284-290.

https://doi.org/10.1016/j.actbio.2015.06.037

[27] Creixell, M., Bohorquez, A.C., Torres-Lugo, M. and Rinaldi, C. (2011) EGFR-Targeted Magnetic Nanoparticle Heaters Kill Cancer Cells without a Perceptible Temperature Rise. ACS Nano, 5, 7124-7129. https://doi.org/10.1021/nn201822b

[28] Pron, G., Mahrour, N., Orlowski, S., Tounekti, O., Poddevin, B., Belehradek, J. and Mir, L.M. (1999) Internalisation of the Bleomycin Molecules Responsible for Bleomycin Toxicity: A Receptor-Mediated Endocytosis Mechanism. Biochemical Pharmacology, 57, 45-56. https://doi.org/10.1016/S0006-2952(98)00282-2

[29] Berg, K., Dietze, A., Kaalhus, O. and Hogset, A. (2005) Site-Specific Drug Delivery by Photochemical Internalization Enhances the Antitumor Effect of Bleomycin. Clinical Cancer Research, 11, 8476-8485. https://doi.org/10.1158/1078-0432.CCR-05-1245

[30] Poddevin, B., Orlowski, S., Belehradek, J. and Mir, L.M. (1991) Very High Cytotoxicity of Bleomycin Introduced into the Cytosol of Cells in Culture. Biochemical Pharmacology, 42, S67-S75. https://doi.org/10.1016/0006-2952(91)90394-K

[31] Mima, H., Yamamoto, S., Ito, M., Tomoshige, R., Tabata, Y., Tamai, K. and Kaneda, Y. (2006) Targeted Chemotherapy against Intraperitoneally Disseminated Colon Carcinoma Using a Cationized Gelatin-Conjugated HVJ Envelope Vector. Molecular Cancer Therapy, 5, 1021-1028. https://doi.org/10.1158/1535-7163.MCT-05-0352

[32] Kawano, H., Komaba, S., Kanamori, T. and Kaneda, Y. (2007) A New Therapy for Highly Effective Tumor Eradication Using HVJ-E Combined with Chemotherapy. BMC Medicine, 5, Article ID: 28. https://doi.org/10.1186/1741-7015-5-28

[33] Voinov, M.A., Pagán, J.O.S., Morrison, E., Smirnova, T.I. and Smirnov, A.I. (2011) Surface-Mediated Production of Hydroxyl Radicals as a Mechanism of Iron Oxide Nanoparticle Biotoxicity. Journal of American Chemical Society, 133, 35-41. https://doi.org/10.1021/ja104683w

[34] Giustini, A.J., Ivkov, R. and Hoopes, P.J. (2011) Magnetic Nanoparticle Biodistribution Following Intratumoral Administration. Nanotechnology, 22, Article ID: 345101. https://doi.org/10.1088/0957-4484/22/34/345101

[35] Takimoto, T., Taylor, G.L., Connaris, H.C., Crennell, S.J. and Portner, A. (2002) Role of the Hemagglutinin-Neuraminidase Protein in the Mechanism of Paramyxovirus-Cell Membrane Fusion. Journal of Virology, 76, 13028-13033. https://doi.org/10.1128/JVI.76.24.13028-13033.2002 